\title{
Radiological surveillance in multiple Endocrine endocrine neoplasia type 1: a double-edged sword?
}

\author{
Ruth Therese Casey', Deborah Saunders², Benjamin George Challis', \\ Deborah Pitfield', Heok Cheow ${ }^{3}$, Ashley Shaw ${ }^{3}$ and Helen Lisa Simpson ${ }^{4}$ \\ ${ }^{1}$ Department of Endocrinology, University of Cambridge, Cambridge Biomedical Research Centre, \\ Addenbrooke's Hospital, Cambridge, UK \\ ${ }^{2}$ East Anglian Regional Radiation Protection Service, Cambridge University Hospitals NHS \\ Foundation Trust, Cambridge, UK \\ ${ }^{3}$ Department of Radiology, Cambridge University Hospitals NHS Foundation Trust, Cambridge, UK \\ ${ }^{4}$ Wolfson Diabetes and Endocrine Clinic, Institute of Metabolic Science, Addenbrooke's Hospital, \\ Cambridge University Hospitals NHS Foundation Trust, Cambridge, UK
}

\author{
Correspondence \\ should be addressed \\ to R Casey \\ Email \\ RC674@medschl.cam.ac.uk
}

\begin{abstract}
Context: Multiple endocrine neoplasia type 1 (MEN1) is a hereditary condition characterised by the predisposition to hyperplasia/tumours of endocrine glands. MEN1related disease, moreover, malignancy related to MEN1, is increasingly responsible for death in up to two-thirds of patients. Although patients undergo radiological and biochemical surveillance, current recommendations for radiological monitoring are based on non-prospective data with little consensus or evidence demonstrating improved outcome from this approach. Here, we sought to determine whether cumulative radiation exposure as part of the recommended radiological screening programme posed a distinct risk in a cohort of patients with MEN1.

Patients and study design: A retrospective review of 43 patients with MEN1 attending our institution between 2007 and 2015 was performed. Demographic and clinical information including phenotype was obtained for all patients. We also obtained details regarding all radiological procedures performed as part of MEN1 surveillance or disease localisation. An estimated effective radiation dose (ED) for each individual patient was calculated. Results: The mean ED for the total patient cohort was $121 \mathrm{mSv}$, and the estimated mean lifetime risk of cancer secondary to radiation exposure was $0.49 \%$. Patients with malignant neuroendocrine tumours (NETS) had significantly higher ED levels compared to patients without metastatic disease $(P<0.0022)$.

Conclusions: In MEN1, radiological surveillance is associated with clinically significant exposure to ionising radiation. In patients with MEN1, multi-modality imaging strategies designed to minimise this exposure should be considered.
\end{abstract}

\section{Introduction}

Multiple endocrine neoplasia type 1 (MEN1) is a hereditary endocrine neoplasia syndrome characterised by autosomal dominant inheritance of mutations in MENIN, a tumour suppressor gene. Patients with MEN1 typically develop tumours or hyperplasia of multiple endocrine glands including the parathyroids, pancreas and pituitary $(1,2,3)$.

Over the past three decades, there has been a paradigm shift in the natural disease course of MEN1, with a reduction in the mortality associated with hormone

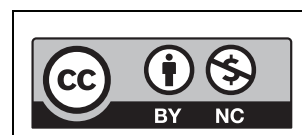

This work is licensed under a Creative Commons Attribution-NonCommercial 4.0 International License.
Endocrine Connections (2017) 6, 151-158 
hypersecretion due to improved surveillance and timely initiation of treatment. Currently, malignant pancreatic NETs and thymic carcinoid tumours are recognised as the most common causes of death in MEN1, respectively. Recent data estimate the mean age at death to be 55 years ( \pm 3 years) with patients having a confirmed diagnosis of MEN1 for a mean duration of 18 years $( \pm 3.2$ years) prior to death (2). As approximately $66 \%$ of patients with MEN1 die as a result of associated malignancy, screening recommendations include annual radiological and biochemical surveillance (3). It is important to note, however, that recommended screening strategies are based on non-prospective data, and current clinical practice guidelines recommend annual surveillance by computed tomography (CT), magnetic resonance imaging (MRI) or endoscopic ultrasound (EUS) (3). Moreover, current guidance advises that local expertise and availability determine the most appropriate imaging modality, thereby highlighting the absence of a consensus regarding the optimum mode and frequency of radiological surveillance (3). It is suggested that thymic and bronchial NETs should be screened for with thoracic and neck CT or MRI every 1-2 years, whereas abdominal CT or MRI should be considered for surveying adrenal glands every three years, and surveillance for pituitary disease by MRI occurs at 3-5 yearly intervals (3). Recently, the European Neuroendocrine Tumour Society (ENETS) published consensus guidance on the management of secretory and non-functioning PNETS (4) and recommend 6-9 monthly CT or MRI for non-functioning PNETS $<2 \mathrm{~cm}$; and monitoring of patients with surgically resected tumours (grade 1-2) every 3-6 months with either modality.

Recent data have demonstrated a 3 -fold increase in the per capita individual radiation exposure from medical diagnostic radiation sources over the past 25 years (5). The majority of evidence supporting ionising radiation as a carcinogen has been derived from epidemiological datasets examining long-term occupational exposure to ionising radiation or follow-up outcome data from survivors of the atomic bomb (6). Ionising radiation can induce cellular damage, referred to as the stochastic effect, without affecting the viability, thereby contributing to carcinogenesis. The probability of tumour induction occurs in proportion to the magnitude of radiation dose exposure $(6,7)$. The effective dose (ED), referred to as cumulative effective dose (CED), quantifies the risk of stochastic effects resulting from exposure to ionising radiation. It has been suggested previously that an ED of $50-100 \mathrm{mSv}$ is the minimum threshold required to promote the development of solid tumours and leukaemia (8). More specifically, data derived from survivors of the atomic bomb have shown that exposure to an ED of $5-125 \mathrm{mSv}$ (mean $29 \mathrm{mSv}$ ) significantly increases the incidence of solid tumours compared with individuals exposed to less than $5 \mathrm{mSv}$ (9). In this study, we undertook a retrospective analysis of 43 patients with MEN1 to determine the amount of ionising radiation exposure during radiological surveillance over an 8-year period and sought to calculate an estimated risk for developing secondary malignancy on the basis of the magnitude of exposure.

\section{Methods}

We performed a retrospective review of medical records of 54 patients attending our institution with a confirmed clinical or genetic diagnosis of MEN1 between the years 2007 and 2015. We obtained clinical and demographic data including, age, sex and clinical phenotype. In addition, we collected data regarding all radiological investigations performed during the study period. Only radiological investigations performed as part of the clinical surveillance strategy for MEN1-associated tumours were included. For example, we did not include radiological investigations performed for an unrelated acute medical problem or other co-existing morbidity. We excluded patients that had radiological investigations performed at other institutions, to maximise the accuracy of the calculated ED. Data from 43 patients were included in the final analysis. As this was a retrospective study of radiological investigations carried out for a clinical indication, and in accordance with best clinical practice, individual patient consent to review historical, anonymised patient-level radiological data was not obtained. However, consent to review patient clinical information was approved by the Cambridge University Hospitals Foundation Trust Research Ethics Committee following ethical review of our research proposal ('The Genomic Analyses of Endocrine and Neuroendocrine tumour study'; REC 14/EE/1059).

\section{Calculation of total effective dose of radiation and lifetime risk}

To calculate the risks from radiation exposure, we undertook an audit of radiological examinations for each individual patient. The imaging datasets for each patient were found using the Picture Archiving Communication http://www.endocrineconnections.org DOI: 10.1530/EC-17-0006
(C) 2017 The authors Published by Bioscientifica Ltd
This work is licensed under a Creative Commons Attribution-NonCommercial 4.0 International License. 
System (PACS) system, which includes details such as modality, anatomical region of imaging and protocol specific to each radiological imaging event. The majority of imaging techniques utilised were either CT or nuclear medicine modalities, and there was marked intra- and inter-individual variability with regards to the radiological protocols used (Supplementary Table 1, see section on supplementary data given at the end of this article). To gain a quantitative indication of the risks from imaging during MEN1 surveillance, the effective dose (ED) was calculated. ED is the sum of tissue-weighted organ equivalent doses. Tissue weighting factors have been derived by the International Commission for Radiological Protection (ICRP), derived from data on incidence of cancers caused by radiation exposure (10).

Computed tomography dose estimations The ED for each CT scan was calculated using the quoted dose-length product (DLP) for each component of the scan protocol and then converting the DLP to ED based on the conversion coefficients published by Shrimpton and coworkers (11). The scan components were broken down into anatomical regions (Supplementary Table 2) as defined by the protocol with the appropriate conversion factor applied to each scan component. The ED for each component was then summed to estimate an overall ED for the entire scan. Lifetime risk of cancer- and age-related risk coefficients reference for each scan was calculated using the total ED and applying an age-related risk coefficient to estimate a percentage lifetime risk of cancer induction for that exposure. The risk for each individual age was estimated by assuming a linear trend between the two coefficients surrounding the patient age at exposure for each scan. However, it was found that the average risk factor was similar to the general risk coefficient of $4.1 \%$ per Sievert (Sv) for adults (11), and this factor was used to estimate the total risks.

Nuclear medicine dose estimations For all patients, examination type and administered activity were recorded for all nuclear medicine scans employed in the localisation of tumours associated with MEN1 including: $\mathrm{TC}^{99} \mathrm{~m}$-sestamibi, Indium-111 pentetreotide single-photon emission computed tomography (SPECT)-CT and Ga ${ }^{68}$. DOTATE PET/CT. The Administration of Radioactive Substances Advisory Committee (ARSAC) guidance notes were used to determine the ED for each patient by linearly extrapolating the ED per maximum permitted activity.
Some patients had $\mathrm{Ga}^{68}$ administrations for which there are no data in the ARSAC guidance notes, and for those patients, the ED per MBq was derived from data reported previously by Hartmann and coworkers (12). The ED for each patient was estimated by summating the individual effective doses from all radiological examinations. The total risk was calculated as described for CT using the general risk coefficient of $4.1 \%$ per Sievert.

\section{Statistical analysis}

We performed all statistical tests with SPSS, version 21.0. Summary statistics calculated included mean and standard deviations for continuous variables and frequencies and percentages for categorical variables. A multiple regression model was used to analyse an association between multiple association variables and the outcome variable of effective dose (ED), and a Mann-Whitney $U$ test was used to compare the means of two distinct patient cohorts.

\section{Results}

\section{Demographics}

Of the 43 patients included in our study, 20 were male and the mean age of the cohort was 52.5 years (22-75 years). The mean duration of disease at the time of review was 14.38 years (2-31 years). Five kindreds were included in the 43 patients analysed in this study (Supplementary Table 1). In kindred 2, with a detected frameshift mutation in MENIN (c.1414delG, p.G472A fs*87), patient \#2 was the proband and patients \#12 and \#13 were diagnosed following predictive genetic testing. In a second kindred (kindred 3), with a confirmed MENIN mutation (c.824G > A and p.R257K), patient \#3 was the index case and patients \#4, 6, 7 and 15 were diagnosed as a result of familial genetic screening. There was insufficient evidence available to ascertain whether members of the remaining three kindreds were diagnosed as part of a family-screening programme or due to a suggestive clinical phenotype.

\section{Clinical characteristics of patient cohort}

The clinical features of the cohort are described in Fig. 1 and Supplementary Table 1. 97.7\% of patients had hyperparathyroidism $(n=42), 76.7 \%(n=33)$ had a PNET, of which $54.5 \%(n=18 / 32)$ were non-functioning and $45.5 \%(n=15 / 33)$ were secretory. Gastrinomas accounted

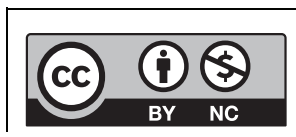

This work is licensed under a Creative Commons Attribution-NonCommercial 4.0 International License. 

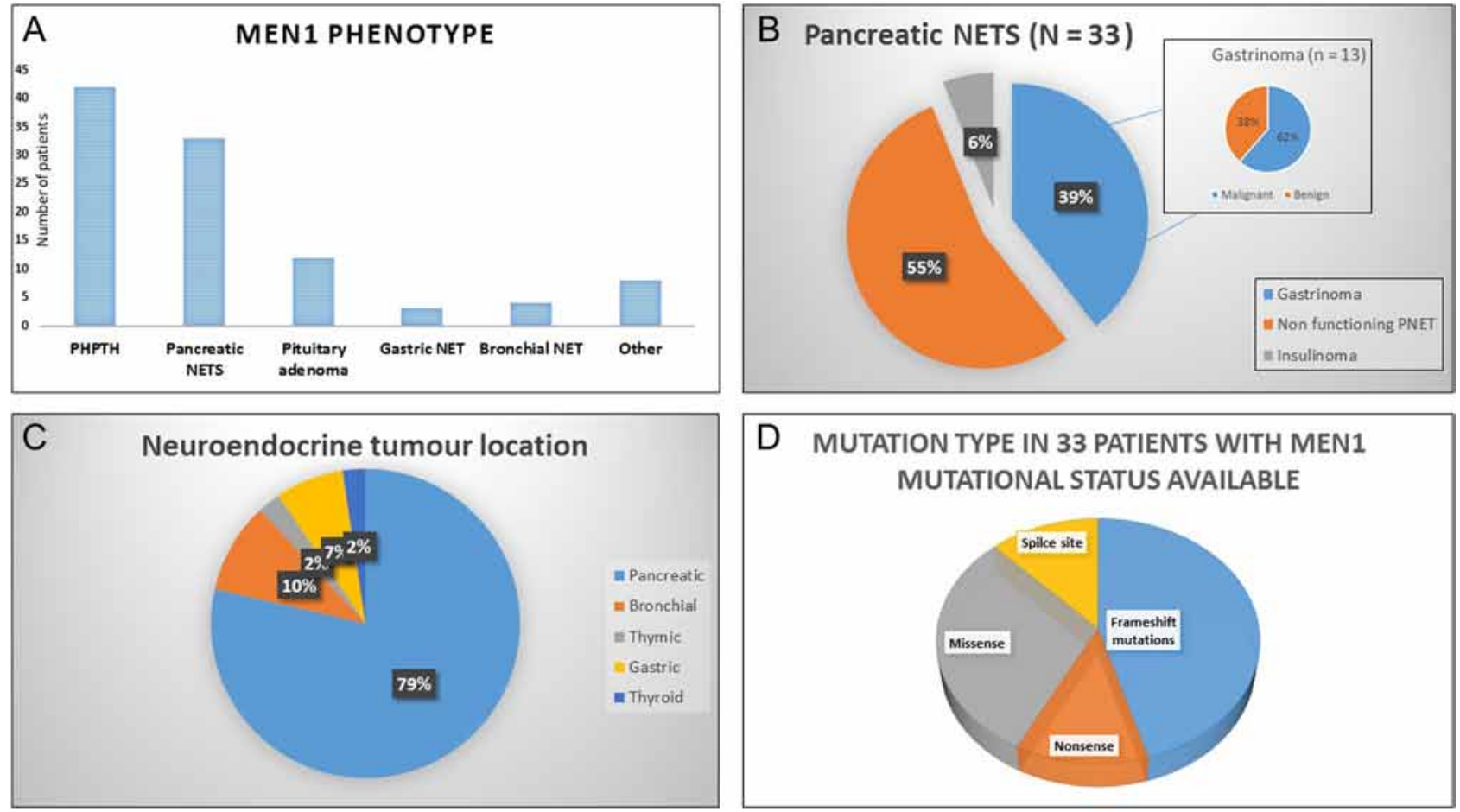

\section{Figure 1}

Clinical phenotype of patients. (A) Demonstrates the MEN1 phenotype observed in this study cohort. (B) Displays the subtypes of pancreatic NETs. (C) Illustrates the neuroendocrine tumour subtypes observed in this study. (D) Demonstrates the spectrum of MENIN mutations identified in this study population.

for the majority of secretory PNETs $(39.4 \%, 13 / 33)$. Pituitary adenomas were present in $27.9 \%(n=12 / 43)$, including 1 (2.3\%) patient with an ACTH-secreting microadenoma and $18.6 \%(n=8 / 43)$ with a prolactinsecreting adenoma. The remaining pituitary adenomas were non-functioning $(n=3 / 43)$.

Less common clinical manifestations included gastric NETs in $7 \%$ of patients $(n=3)$, bronchial NET in $9.3 \%(n=4)$, thymic NET in $2.3 \%(n=1)$, thyroid NET in $2.3 \%(n=1)$, adrenal adenomas in $11.6 \%(n=5)$ and adrenocortical carcinoma in $2.3 \% \quad(n=1)$. A diagnosis of metastatic disease from all-cause NET was made in $30.2 \%$ of patients $(n=13 / 43)$ with the liver being the most common site for distant metastases. Gastrinomas were the most common NET to metastasise in this cohort with $18.6 \%(8 / 43)$ identified with metastatic disease during the study period.

Genetic confirmation of the specific MENIN gene mutations was available for $33 / 43$ patients $(76.7 \%)$. The following mutations were identified: frameshift $(45.5 \%$, $n=15)$, nonsense $(12.1 \%, n=4)$, missense $(30.3 \% ; n=10)$ and splice site $(12.1 \% ; n=4)$. One patient died during the study period. No patients developed a 'non-MEN1'recognised tumour or haematological malignancy during the study period.

\section{Radiation exposure}

The mean effective dose (ED) calculated for radiation exposure from CT imaging or surveillance alone was $109 \mathrm{mSv}$, and the mean overall ED, including nuclear imaging modalities, was $121 \mathrm{mSv}$. The maximum ED

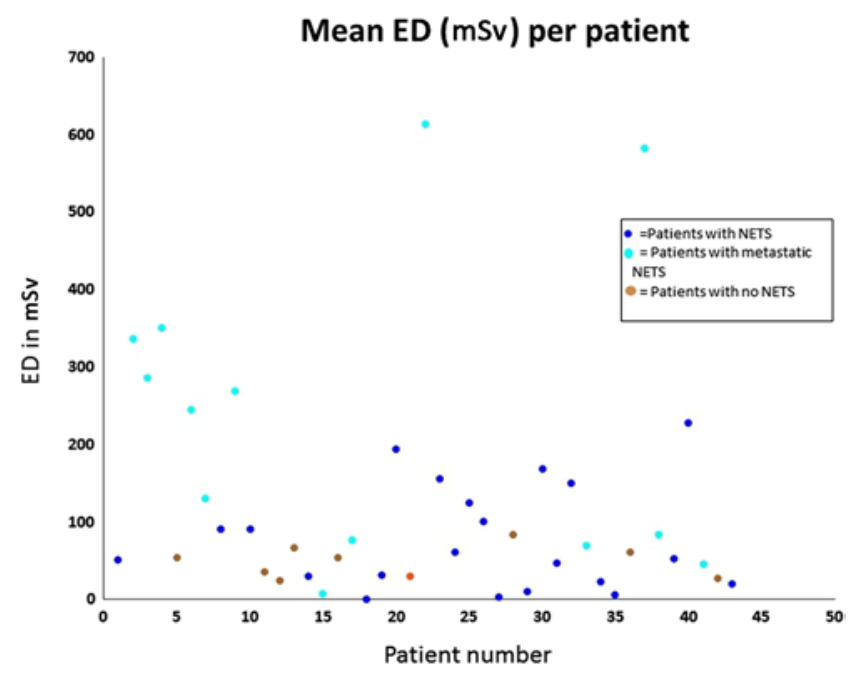

Figure 2

Mean effective dose (ED) per individual patient.

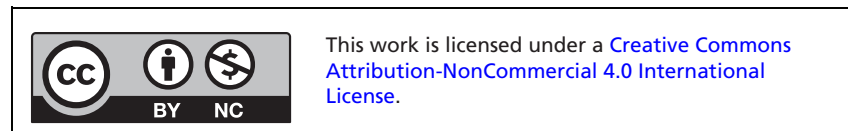


Table 1 Calculated mean effective dose (ED) and percentage lifetime risk for cohort categorised by the presence of NET.

\begin{tabular}{lccc}
\hline Patient \# & Mean effective dose $(\mathrm{mSV})$ & Mean \% lifetime risk \\
\cline { 1 - 1 } \cline { 3 - 3 } \#1 & 51.8 & 0.21 \\
\#8 & 91.0 & 0.37 \\
\#10 & 91.3 & 0.37 \\
\#14 & 30.4 & 0.12 \\
\#18 & 0.3 & 0.00 \\
\#19 & 32.1 & 0.13 \\
\#20 & 194.4 & 0.80 \\
\#23 & 155.7 & 0.64 \\
\#24 & 62.0 & 0.25 \\
\#25 & 124.5 & 0.51 \\
\#26 & 100.7 & 0.41 \\
\#27 & 3.5 & 0.01 \\
\#29 & 9.9 & 0.04 \\
\#30 & 168.8 & 0.69 \\
\#31 & 47.5 & 0.19 \\
\#32 & 150.3 & 0.62 \\
\#34 & 23.2 & 0.10 \\
\#35 & 6.2 & 0.03 \\
\#39 & 52.9 & 0.22 \\
\#40 & 228.7 & 0.94 \\
\#43 & 21.0 & 0.09 \\
& &
\end{tabular}

observed was $613 \mathrm{mSv}$ (Fig. 2 and Tables 1, 2, 3). A mean percentage lifetime risk of cancer secondary to radiation exposure was calculated for this cohort over the eightyear study period. Over this interval, the mean percentage lifetime risk of developing a secondary malignancy was $0.49 \%$. In one individual, the maximum additional lifetime risk of a cancer related to radiation exposure observed was $2.51 \%$.

\section{Factors associated with high radiation exposure}

The mean ED for patients with metastatic disease was $227.8 \mathrm{mSV}$ (median 188.2) vs $141.6 \mathrm{mSV}$ (median 58.3) for

Table 2 Calculated mean effective dose (ED) and percentage lifetime risk for cohort categorised by metastatic NET.

\begin{tabular}{|c|c|c|}
\hline Patient & Mean effective dose (mSV) & Mean $\%$ lifetime risk \\
\hline$\# 2$ & 335.6 & 1.38 \\
\hline \#3 & 286.9 & 1.18 \\
\hline \#4 & 350.8 & 1.44 \\
\hline \#6 & 245.6 & 1.01 \\
\hline$\# 7$ & 130.8 & 0.54 \\
\hline \#9 & 269.1 & 1.10 \\
\hline \#15 & 7.7 & 0.03 \\
\hline \#17 & 76.7 & 0.31 \\
\hline \#22 & 613.3 & 2.51 \\
\hline \#33 & 70.4 & 0.29 \\
\hline \#37 & 581.9 & 2.39 \\
\hline \#38 & 84.1 & 0.34 \\
\hline \#41 & 46.4 & 0.19 \\
\hline
\end{tabular}

Table 3 Calculated mean effective dose (ED) and percentage lifetime risk for cohort categorised by no NET.

\begin{tabular}{|c|c|c|}
\hline Patient & Mean effective dose (mSV) & Mean \% lifetime risk \\
\hline \#5 & 55.8 & 0.23 \\
\hline \#11 & 36.7 & 0.15 \\
\hline \#12 & 25.2 & 0.10 \\
\hline \#13 & 68.0 & 0.28 \\
\hline \#16 & 54.5 & 0.22 \\
\hline \#21 & 30.3 & 0.12 \\
\hline \#28 & 84.2 & 0.35 \\
\hline \#36 & 62.1 & 0.25 \\
\hline \#42 & 28.4 & 0.12 \\
\hline
\end{tabular}

patients without metastatic disease. Statistically, the ED was significantly higher in patients with metastatic disease compared with patients without metastases (Z-score-3.05, $P<0.0022)$. ED was not significantly higher in patients with PNETs compared to those subjects without PNETS ( $Z$-score $1.1, P=0.27$ ). The mean ED in patients with a functioning PNET versus patients with a non-functioning PNET was $191.7 \mathrm{mSV}$ (median 155.7) vs $98.5 \mathrm{mSV}$ (median 76.7), but this did not reach statistical significance ( $Z$-score -1.56, $P=0.058)$. No significant difference in ED values was detected for patients with pituitary disease, bronchial, gastric or thymic NETs. Finally, we found no significant difference in ED between patients with a specific mutation in the MENIN gene ( $Z$-score: $-1.11, P=0.267)$.

\section{Discussion}

This is the first study to investigate the ED of ionising radiation received by a cohort of MEN1 patients. In patients with radiation exposure over a protracted period, an estimated threshold of $50-100 \mathrm{mSv}$ has been associated with a significantly increased risk of developing secondary solid tumours and/or leukaemia, based on available epidemiological data (8). A mean ED of $121 \mathrm{mSv}$ was observed in this cohort and $65.1 \%(28 / 43)$ of our cohort had a mean ED $>50 \mathrm{mSv}$, with $35 \%(15 / 43)$ having a mean ED $>100 \mathrm{mSv}$ (Fig. 2 and Tables 1, 2, 3). The ED reported for patients in this study is based on an eight-year study period. Ito and coworkers (2) reported that patients with MEN1 had a mean duration of diagnosis of 18 years prior to death. This suggests that, on average, patients included in our study will have ten additional years of radiological surveillance and, therefore, increased lifetime radiation exposure over that reported in this study. The maximum individual mean ED was $613 \mathrm{mSv}$, calculated in a 45-yearold gentleman with metastatic gastric carcinoid who was undergoing biannual surveillance CT examinations

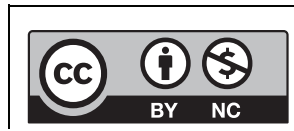

This work is licensed under a Creative Commons Attribution-NonCommercial 4.0 International License. 
to assess for disease progression. We also found that metastatic disease and the presence of functioning PNETs were associated with significantly higher mean ED levels, reflecting adoption of a more aggressive surveillance strategy for these patients. The rationale for an aggressive surveillance approach is based on the presumption that early pre-symptomatic detection of neoplasia associated with MEN1 may reduce the associated mortality (3). However, currently, there are no data to support the notion that more aggressive radiological surveillance leads to improved survival outcomes for this patient cohort, and further prospective data are required.

It has been estimated that exposure to ionising radiation for diagnostic purposes may account for $2 \%$ of all cancers worldwide (13). The ED in other patient groups requiring long-term radiological follow-up has been reported previously. For example, Chatu and coworkers (6) undertook a systematic review to investigate the ED received by over 1700 patients with inflammatory bowel disease and found that $8.4 \%$ of subjects had an ED exposure greater than $50 \mathrm{mSv}$, which is substantially less than the $65.1 \%$ of subjects with ED greater than $50 \mathrm{mSv}$ in our study. Similarly, in dialysis patients awaiting renal transplant, De Mauri and coworkers found that $43 \%$ of patients had an ED of $>50 \mathrm{mSv}$ per year (14).

The effect of repeated exposure to diagnostic doses of ionising radiation in patients with a pre-existing cancer susceptibility syndrome has not been well studied. There is evidence to suggest that patients with mutations in genes involved in DNA repair or tumour suppression may be more sensitive to the effects of therapeutic radiation (15). Therefore, there is a theoretical risk, at least, that linear, repeated exposure to ionising radiation in patients with a hereditary defect in a tumour suppressor gene, such as MENIN, increases the oncogenic risk.

The Menin protein interacts with several binding partners, which play integral roles in gene transcription, gene stability and cellular division (16, 17). Recently, Albers and coworkers identified a genotype-phenotype correlation between mutations in MENIN affecting codons 428-610, and the development of functional PNETS, aggressive or metastatic PNETs and mortality associated with PNETS (16). A possible explanation for these findings is that mutations affecting these codons prevented a functional interaction with CHES1, a forkhead transcription factor that functions as a checkpoint suppressor and is activated in response to DNA damage (18). As CHES1 plays an important role in gene transcription and DNA repair, it was hypothesised that

http://www.endocrineconnections.org DOI: 10.1530/EC-17-0006

(c) 2017 The authors Published by Bioscientifica Ltd loss of interaction with CHES1 may result in uncontrolled cell division and a more aggressive phenotype (18). In addition, loss of the CHES1 interaction may also potentiate the effects of radiation exposure, as the in vitro interaction of Menin with CHES1 has been demonstrated to be important for the homology-directed repair of DNA damage (19). In our study, although $14 \%(n=6)$ of subjects were found to have mutations affecting codons 428-610, these specific patients were not subjected to higher ED and did not demonstrate increased risk of malignant disease (data not shown). Nevertheless, it is recognised that the consequences of MENIN mutations are likely to disrupt protein interactions that are critical to cell cycle regulation. We found, in agreement with other studies (17), that the most common type of mutation in MEN1 are frameshift mutations, which result in a truncated protein that may affect protein-protein interactions and/or result in nonsense-mediated RNA decay of the translated protein (20).

It is notable that two patients were found to have breast cancer, which may be a MEN1-related tumour (21), but no other solid tumours or haematological malignancies not normally associated with MEN1 were identified. Although it is appreciated that probability of tumour induction increases in a linear fashion (11) in proportion to radiation dose, the interval required for tumour induction is not well understood. Therefore, the discordance between the high mean ED identified in this cohort and the lack of secondary tumour induction, may be accounted for by the relatively short review period of this study (8 years).

We recognise the limitations of this study that include retrospective design, short study period, exclusion of radiological investigations performed at other institutions (which would underestimate ED) and incomplete data regarding mutational status for 11 patients. In future, prospective studies with long follow-up will be required to determine the true effect of linear radiation exposure on the risk of secondary malignancy in patients with MEN1.

The optimal radiological surveillance strategy in MEN1 remains unclear. Cross-sectional imaging is important for early detection and subsequent treatment of neuroendocrine tumours in patients with MEN1. The results reported from this study serve as a reminder of the risks associated with cumulative exposure to ionising radiation during long-term radiological follow-up. We observed a mean cohort ED of $121 \mathrm{mSv}$ over an 8-year study period, which far exceeds levels of radiation exposure deemed safe. Whether this exposure poses significant 
clinical risk requires further study. Nevertheless, our findings should be considered in the context of future radiological surveillance protocols developed for inherited tumour syndromes including those related to multiple endocrine neoplasia, succinate dehydrogenase gene mutations $(S D H)$ and Von Hipple-Lindau (VHL) disease.

Although there are limited data on the effect of radiation exposure in hereditary cancer syndromes, we suggest that a multi-modality approach utilising CT/MRI/EUS should be adopted in a MEN1 cohort to minimise the radiation exposure and associated effects. More prospective data are required to establish the risks of developing malignancy related to radiation exposure, to tailor bespoke screening programmes. Ongoing prospective collection of data will also establish whether genotype-phenotype correlations exist and guide tailored radiological surveillance. Furthermore, our study highlights the need for further investigation of the cellular, biological effects of radiation in patient cohorts with known hereditary tumour susceptibility. In conclusion, we recommend better consideration of risks of radiation dose when managing patients with hereditary neoplasia syndromes.

\section{Supplementary data}

This is linked to the online version of the paper at http://dx.doi.org/10.1530/ EC-17-0006.

\section{Declaration of interest}

The authors declare that there is no conflict of interest that could be perceived as prejudicing the impartiality of the research reported.

\section{Funding}

Dr Ruth Casey received a bursary from Sanofi in June 2015 and Dr Ashley Shaw receives funding from the Cambridge Biomedical Research Council.

\section{Author contribution statement}

Dr Ruth Casey, Dr Ben Challis, Deborah Pitfield and Deborah Saunders were involved in data collection and interpretation. Dr Shaw, Dr Cheow and Dr Simpson were involved in study design and data interpretation. All authors contributed to manuscript preparation and the final review of the manuscript.

\section{References}

1 Dreijerink KM, Höppener JW, Timmers HM \& Lips CJ. Mechanisms of disease: multiple endocrine neoplasia type 1-relation to chromatin modifications and transcription regulation. Nature Clinical Practice Endocrinology and Metabolism 20062 562-570. (doi:10.1038/ ncpendmet0292)

2 Ito T, Igarashi H, Uehara H, Berna MJ \& Jensen RT. Causes of death and prognostic factors in multiple endocrine neoplasia type 1 : a prospective study: comparison of 106 MEN1/Zollinger-Ellison syndrome patients with 1613 literature MEN1 patients with or without pancreatic endocrine tumors. Medicine 201392 135-181. (doi:10.1097/md.0b013e3182954af1)

3 Thakker RV, Newey PJ, Walls GV, Bilezikian J, Dralle H, Ebeling PR, Melmed S, Sakurai A, Tonelli F \& Brandi ML. Clinical practice guidelines for multiple endocrine neoplasia type 1 (MEN1). Journal of Clinical Endocrinology and Metabolism 201297 2990-3011. (doi:10.1210/jc.2012-1230)

4 Falconi M, Eriksson B, Kaltsas G, Bartsch DK, Capdevila J, Caplin M, Kos-Kudla B, Kwekkeboom D, Rindi G, Klöppel G, et al. ENETS consensus guidelines update for the management of patients with functional pancreatic neuroendocrine tumors and non-functional pancreatic neuroendocrine tumors. Neuroendocrinology 2016103 153-171. (doi:10.1159/000443171)

5 Administration of Radioactive Substances Advisory Committee. Notes for Guidance on the Clinical Administration of Radiopharmaceuticals and Use of Sealed Radioactive Sources, December 1998. Didcot, Oxfordshire, UK: ARSAC, National Radiological Protection Board. (available at: http://webarchive.nationalarchives.gov. uk/20050513213558/http://advisorybodies.doh.gov.uk/arsac/nfgdec1998.pdf)

6 International Commission on Radiological Protection. The 2007 Recommendations of the International Commission on Radiological Protection. ICRP Publication 103. Annals of the ICRP 37 (2-4). (available at: http://www.icrp.org/publication.asp?id=ICRP\%20 Publication\%20103)

7 Wall BF, Haylock R, Jansen JTM, Hillier MC, Hart D \& Shrimpton PC. Radiation risks from medical X-ray examinations as a function of the age and sex of the patient. HPA-CRCE-028. Didcot, Oxfordshire, UK: Health Protection Agency, 2011. (available at: https://www.gov.uk/ government/uploads/system/uploads/attachment_data/file/340147/ HPA-CRCE-028_for_website.pdf).

8 Brenner DJ, Doll R, Goodhead DT, Hall EJ, Land CE, Little JB, Lubin JH, Preston DL, Preston RJ, Puskin JS, et al. Cancer risks attributable to low doses of ionizing radiation: assessing what we really know. PNAS 2003 100 13761-13766. (doi:10.1073/pnas.2235592100)

9 Pierce DA \& Preston DL. Radiation-related cancer risks at low doses among atomic bomb survivors. Radiation Research 2000154 178-186. (doi:10.1667/0033-7587(2000)154[0178:RRCRAL]2.0.CO;2)

10 Chatu S, Subramanian V \& Pollok RC. Meta-analysis: diagnostic medical radiation exposure in inflammatory bowel disease. Alimentary Pharmacology and Therapeutics 201235 529-539. (doi:10.1111/j.13652036.2011.04975.x)

11 Shrimpton PC, Kansen J \& Harrison J. Updated estimates of typical effective doses for common CT examinations in the UK following the 2011 national review. British Journal of Radiology 20168920150346. (doi:10.1259/bjr.20150346)

12 Hartmann H, Zöphel K, Freudenberg R, Oehme L, Andreeff M, Wunderlich G, Eisenhofer G \& Kotzerke J. Radiation exposure of patients during 68Ga-DOTATOC PET/CT examinations. Nuklearmedizin 200948 201-207.

13 Flasar M \& Patil S. Radiating disparity in IBD. Digestive Diseases and Sciences 201459 504-506. (doi:10.1007/s10620-013-2922-4)

14 De Mauri A, Matheoud R, Carriero A, Lizio D, Chiarinotti D $\&$ Brambilla M. Radiation exposure from medical imaging in dialyzed patients undergoing renal pre-transplant evaluation. Journal of Nephrology 201630 141-146. (doi:10.1007/s40620-0160275-8)

15 Allan JM. Genetic susceptibility to radiogenic cancer in humans. Health Physics 200895 677-686. (doi:10.1097/01. HP.0000326339.06405.ea)

16 Albers M, Knoop R, Chaloupka B, Lopez CL, Fendrich V, Kann PH \& Waldmann J. Higher risk of aggressive pancreatic neuroendocrine tumors in MEN1 patients with MEN1 mutations affecting the CHES1 interacting MENIN domain. Journal of Clinical

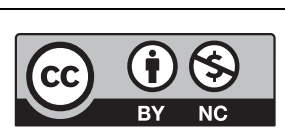

This work is licensed under a Creative Commons Attribution-NonCommercial 4.0 International License. 
Endocrinology and Metabolism 201499 E2387-E2391. (doi:10.1210/ jc.2013-4432)

17 Lemos MC \& Thakker RV. Multiple endocrine neoplasia type 1 (MEN1): analysis of 1336 mutations reported in the first decade following identification of the gene. Human Mutation 200829 22-32. (doi:10.1002/humu.20605)

18 Huot G, Vernier M, Bourdeau V, Doucet L, Saint-Germain E, Gaumont-Leclerc MF, Moro A \& Ferbeyre G. CHES1/FOXN3 regulates cell proliferation by repressing PIM2 and protein biosynthesis. Molecular Biology of the Cell 201425 554-565. (doi:10.1091/mbc.E1302-0110)
19 Gallo A, Agnese S, Esposito I, Galgani M \& Avvedimento VE. Menin stimulates homology-directed DNA repair. FEBS Letters $2010 \mathbf{5 8 4}$ 4531-4536. (doi:10.1016/j.febslet.2010.10.032)

20 Kuzmiak HA \& Maquat LE. Applying nonsense-mediated mRNA decay research to the clinic: progress and challenges. Trends in Molecular Medicine 200612 306-316. (doi:10.1016/j.molmed.2006.05.005)

21 Koen MA, Dreijerink MD, Goudet P, Burgess JR, Valk GD \& International Breast Cancer in MEN1 Study Group. Breast-cancer predisposition in multiple endocrine neoplasia type 1. New England Journal of Medicine 2014371 583-584. (doi:10.1056/ NEJMc1406028)

Received in final form 12 January 2017

Accepted 27 February 2017
This work is licensed under a Creative Commons Attribution-NonCommercial 4.0 International License. 